\title{
Examining spondyloarthritis in patients with inflammatory bowel disease
}

\author{
Catherine Burns ${ }^{1}$, Reem Jan² \\ ${ }^{1}$ Indiana University School of Medicine, ${ }^{2}$ University of Chicago Department of Internal Medicine, \\ Section of Rheumatology
}

Background/Objective: Axial spondyloarthritis refers to a syndrome of inflammatory back pain associated with radiographic or magnetic resonance imaging abnormalities. Peripheral spondyloarthritis can include dactylitis, enthesitis or oligo-arthritis. Together these encompass common extra-gastrointestinal manifestations of inflammatory bowel disease (IBD), with the prevalence of ankylosing spondylitis estimated to be about 3\% in patients with IBD and unspecified sacroiliitis occurring in $10 \%$. The goals of this study are to validate the accuracy of the Toronto Axial Spondyloarthritis Questionnaire in identifying patients with rheumatologic symptoms in the context of IBD, to re-evaluate the prevalence of spondyloarthritis in this population, and to identify any differences in referral rate between racial and ethnic groups.

Methods: Patients were selected based on the following criteria: diagnosis of IBD, upcoming appointment with their gastroenterologist, prior consent in the Genesys database and access to an operational MyChart account. Patients were sent a link and asked to complete a RedCap survey modified from the Toronto Axial Spondyloarthritis Questionnaire. Positive patient responses will be analyzed in a follow-up visit with a rheumatologist to investigate the patients' symptoms.

Results: At present, the survey was sent to 86 patients with 26 respondents completing the survey. Of the respondents, 6 patients had experienced back pain for a duration of 3 months or longer. Eleven patients had pain and swelling of a joint unrelated to injury. Seven patients experienced pain and swelling of an entire finger or toe unrelated to injury. Seven patients experienced heel pain unrelated to injury. One patient is followed by rheumatology.

Conclusion/Potential Impact: Major extra gastrointestinal manifestations of IBD include axial or peripheral spondyloarthritis which can lead to disabling back pain and/ or joint disease. With the modified Toronto Axial Spondyloarthritis Questionnaire, gastroenterologists could have a better way to identify concerning symptoms, leading to increased referral to rheumatologists and potential changes in treatment plans. 